\title{
Inflammatory and coagulatory markers and exposure to different size fractions of particle mass, number and surface area air concentrations in Swedish iron foundries, in particular respirable quartz
}

\author{
Håkan Westberg ${ }^{1,2,3,6} \cdot$ Alexander Hedbrant ${ }^{2,3} \cdot$ Alexander Persson $^{2,3} \cdot$ Ing-Liss Bryngelsson ${ }^{1} \cdot$ Anders Johansson $^{1}$. \\ Annette Ericsson ${ }^{1}$ - Bengt Sjögren ${ }^{4}$ - Leo Stockfelt ${ }^{5}$. Eva Särndahl ${ }^{2,3}$ • Lena Andersson ${ }^{1,2}$
}

Received: 17 April 2018 / Accepted: 28 May 2019 / Published online: 4 June 2019

(c) The Author(s) 2019

\begin{abstract}
Purpose To study the relationship between inhalation of airborne particles and quartz in Swedish iron foundries and markers of inflammation and coagulation in blood.

Methods Personal sampling of respirable dust and quartz was performed for 85 subjects in three Swedish iron foundries. Stationary measurements were used to study the concentrations of respirable dust and quartz, inhalable and total dust, PM10 and PM2.5, as well as the particle surface area and the particle number concentrations. Markers of inflammation, namely interleukins (IL-1 $\beta$, IL-6, IL-8, IL-10 and IL-12), C-reactive protein, and serum amyloid A (SAA) were measured in plasma or serum, together with markers of coagulation including fibrinogen, factor VIII (FVIII), von Willebrand factor and D-dimer. Complete sampling was performed on the second or third day of a working week after a work-free weekend, and follow-up samples were collected 2 days later. A mixed model analysis was performed including sex, age, smoking, infections, blood group, sampling day and BMI as covariates.

Results The average 8-h time-weighted average air concentrations of respirable dust and quartz were $0.85 \mathrm{mg} / \mathrm{m}^{3}$ and $0.052 \mathrm{mg} / \mathrm{m}^{3}$, respectively. Participants in high-exposure groups with respect to some of the measured particle types exhibited significantly elevated levels of SAA, fibrinogen and FVIII.

Conclusions These observed relationships between particle exposure and inflammatory markers may indicate an increased risk of cardiovascular disease among foundry workers with high particulate exposure.
\end{abstract}

Keywords Inflammatory markers $\cdot$ Particle mass $\cdot$ Particle number $\cdot$ Particle surface area $\cdot$ Respirable quartz $\cdot$ Iron foundries

Håkan Westberg

hakan.westberg@regionorebrolan.se

1 Department of Occupational and Environmental Medicine, Faculty of Medicine and Health, Örebro University, 70182 Örebro, Sweden

2 Department of Medical Sciences, School of Medicine and Health, Örebro University, 70182 Örebro, Sweden

3 Inflammatory Response and Infection Susceptibility Centre (iRiSC), Örebro University, 70182 Örebro, Sweden

4 Work Environment Toxicology, Institute of Environmental Medicine, Karolinska Institutet, 17177 Stockholm, Sweden

5 Department of Occupational and Environmental Medicine, University of Gothenburg, PB 414, 40530 Göteborg, Sweden

6 Department of Occupational and Environmental Medicine, Örebro University Hospital, 70185 Örebro, Sweden

\section{Introduction}

In 2016, Swedish foundries produced 292,000 tons, of which 209,000 tons were from the iron foundries that collectively employ 7500 foundry workers (Nayström 2018). In the foundry industry, concerns about aerosol exposure have primarily related to respirable quartz (CAS no.1408060-7), mostly due the risk of silicosis (Rees and Weiner 1994; Rosenman et al. 1996; Schudel 1960) and lung cancer (Sherson et al. 1991; Andjelkovich et al. 1990; Rodriguez et al. 2000; Westberg and Bellander 2003; Xu et al. 1996). The working environment of iron foundries has also been linked to elevated risk of cardiovascular diseases (Koskela et al. 2005; Palda 2003; Fan et al. 2018) and mortality (Vena 
et al. 1985; Decoufle and Wood 1979; Silverstein et al. 1986; Yoon and Hahn 2014).

Exposure to ambient airborne particulate matter inhalation has been associated with increases in mortality and hospital admissions due to cardiovascular diseases, and mechanistic evidence from animal and human studies suggests that these outcomes are associated with systemic inflammatory responses. Other potentially relevant processes include disturbances in the autonomic nervous system, and the direct effects of particles reaching the systemic circulation are other potential pathways (Brook et al. 2010).

The inflammatory causal link between inhalation of particulate matter and cardiovascular disease was first proposed in the mid-1990s (Seaton et al.1995; Sjögren 1997). Later publications revealed some of the mechanisms underpinning this link, notably the formation of the NLRP3 inflammasome upon exposure of intracellular receptors to particles, which leads to the activation of the enzyme caspase-1, and subsequently of interleukin IL-1 $\beta$ and other cytokines (Dagenais et al. 2012; Dostert et al.2008; Peeters et al. 2013).

Several inflammatory markers, including IL-6, C-reactive protein (CRP), and fibrinogen, are established risk factors for cardiovascular disease (Danesh et al. 2000, 2005, 2008). Serum amyloid A (SAA) has also been proposed as a risk factor for cardiovascular disease as a component of a battery of several biomarkers (Arant et al. 2009). Quartz exposure has been linked to increased levels of TNF- $\alpha$, IL- 6 and IL-1 $\beta$, according to a review of animal studies (Kawasaki 2015). In a series of studies, we have determined exposure responses for various inflammatory markers with respect to particle exposure in mechanical and welding workshops, cement plants, aluminium foundries and in the pulp and paper industry (Ohlson et al. 2010; Westberg et al. 2016). Here, we extend this work to cover iron foundries.

The purpose of this study was to investigate a possible exposure-response relationship between particulate exposure (quantified in terms of levels of respirable dust and quartz, and particle mass, number and surface area concentrations in air) and markers of inflammation and coagulation.

\section{Methods}

\section{Study group}

The study was performed at three Swedish iron foundriesa large foundry employing 440 workers and two smaller ones employing 90 and 25 workers, respectively. The yearly outputs of these foundries amount to 100,000, 25,000 and 2000 tons of castings, respectively. These castings are mainly based on iron and grey iron alloys, and include products such as motor heads and blocks for trucks, large components for the wind power industry and medium- and small-sized lego castings. The jobs and departments included in the study were sand preparation, core making, moulding, casting, shake-out operations, fettling, inspection, maintenance and repair. The sand used for moulding was mostly green sand (75\% $\mathrm{SiO}_{2}, 6 \%$ carbon black, 5-6\% bentonite and $13-14 \%$ water), but chemical binders such as furan (furfuryl alcohol, urea, phenol, formaldehyde, $p$-toluenesulphonic acid or phosphoric acid) and silicate ester (sodium meta silicate and organic ester) were added to the silica sand. The tasks performed at the foundries are mechanized and manual moulding and casting. The most commonly used core binder was epoxy- $\mathrm{SO}_{2}$ and sodium silicate, but coldbox (isocyanateMDI, polyol, phenol formaldehyde) resin with an amine as catalytic agent was also used for this purpose (American Foundry Society 2018). Eighty-five foundry workers (80 men and 5 women), most of whom worked day shifts, participated in the study. Their ages ranged from 21 to 67 years, with a mean of 43 , and the participants were evenly distributed over 10-year age groups. They had worked in the same workplace for 12 years on average, with a maximum of 45 years' continuous employment. Eighty-three participants reported their smoking habits: 17 were current smokers, 18 ex-smokers and 48 were never smokers.

\section{Study design}

Six separate 4-day measurement campaigns were conducted between March 2015 and September 2016. During each campaign, aerosol and blood sampling was performed on the second or third working day after a work-free weekend. Blood sampling was performed twice for each subject, at work: once in the afternoon (3-4.30 p.m.) on the first day of the campaign, and again 2 days later (i.e., on day 4 or 5). The post-shift sampling design was intended to control for circadian variation. All participants completed a questionnaire containing items relating to their current and previous working conditions, height and weight, smoking habits, medication, symptoms of respiratory irritation and infections or other inflammatory conditions that could affect the levels of the studied biomarkers. All participants were at work during our investigation. Seven participants reported chronic diseases such as thyroid imbalance, type 2 diabetes, scoliosis, hypertension, depression, asthma or COPD.

\section{Aerosol measurements and quartz analysis}

The respiratory tract comprises three regions: the extrathoracic region (including the nose and throat), the thoracic region and the alveolar region. Particles that enter the extrathoracic region constitute the inhalable fraction, those that penetrate into the thoracic region constitute the thoracic fraction and particles deposited into the alveolar region constitute the respirable fraction. The $\mathrm{PM}_{10}$ and $\mathrm{PM}_{2.5}$ fractions, 
which are discussed widely in environmental analyses, correspond to the thoracic and respirable fractions, respectively (Vincent 2005).

The participants' respirable and quartz dust exposure was determined by personal sampling on the 2nd or 3rd day after a work-free weekend, on the same day as the first blood sample collection. Personal sampling of respirable dust was performed by collecting 8-h full shift samples according to standard protocols using GSP samplers operating at $3.5 \mathrm{l} /$ min (HSE 2000). A minority of samples were collected using SKC AircheckXR5000 or GSA SG5100 sampling pumps. Respirable crystalline quartz and cristobalite were determined by X-ray diffraction (NIOSH 1994a) (modified).

Stationary measurements (area measurements) were acquired using measuring rigs. These measurements were conducted in the departments where the participants worked during their shifts and included sampling of respirable dust and quartz using the same techniques as for the personal samples. Total dust was determined by collecting 8 -h full shift samples according to a modified gravimetric method (NIOSH 1994b) using open-faced $25 \mathrm{~mm}$ filter cassettes (OFC) with an air flow of $2.0 \mathrm{l} / \mathrm{min}$. Respirable dust was determined using SKC HD aluminium cyclones with $25 \mathrm{~mm}$ filters, operating at an air flow of $2.5 \mathrm{l} / \mathrm{min}$ (HSE 2000). Air concentrations of $\mathrm{PM}_{10}$ and $\mathrm{PM}_{2.5}$ were determined using a ChemPass system operating at $1.8 \mathrm{l} / \mathrm{min}$ (EN 1999, 2005). For $\mathrm{PM}_{1}$, a Dusttrak DRX Monitor 8533 was used. Particle surface area air concentrations were determined using an A-trak 9000 (Aero Trak Nanoparticle Aerosol Monitor) instrument, which determines the particle surface area for particles in the size range $10-1000 \mathrm{~nm}$ in real time. Particle number concentrations were measured based on particles in the $20-1000 \mathrm{~nm}$ size range, using a real-time aerosol monitoring instrument (P-Trak 8525 Ultrafine Particle Counter).

\section{Markers of inflammation and coagulation}

The blood samples were centrifuged and stored at $-70^{\circ}$ in a biobank (Dnr 13OLL718-5). The concentrations of several interleukins (IL-1 $\beta$, IL-6, IL-8, IL-10 and IL-12) and tumor necrosis factor (TNF) were determined at the research laboratory of iRiSC, Örebro University. These measurements were performed with a Luminex 200TM recorder (12212 Technology Blvd, Austin, Texas, USA) and analyzed using the Luminex XPONENT software package. High-sensitivity CRP, fibrinogen, D-dimer and blood groups (ABO) were determined at the Clinical Research Center, Örebro University Hospital. Serum amyloid A (SAA), factor VIII (FVIII) and the von Willebrand factor (vWF) were analysed at the Department of Occupational and Environmental Medicine, Sahlgrenska University Hospital, Göteborg.

\section{Exposure measures}

Exposure was assessed by performing personal sampling to measure concentrations of respirable dust and quartz, and by performing stationary measurements in each department that served as proxies for the total and inhalable dust, $\mathrm{PM}_{10}, \mathrm{PM}_{2.5}, \mathrm{PM}_{1}$, particle surface area and particle number concentrations in the air. The 28 participants working in known high-exposure jobs (particularly shake-out and fettling operations) wore respirators while working. To account for the respirators' effects, the raw measurements for these subjects were adjusted to better assess their true exposure. Specifically, it was assumed that these workers experienced zero exposure while wearing a respirator mask; their exposure during the remainder of their shifts was estimated based on either personal sampling or the measured background concentrations for the departments they worked in while not wearing respirators.

Three exposure measures were computed: the nonadjusted exposure ( $\left.C_{\text {average }}\right)$ as determined by personal sampling; the average adjusted ( $\left.C_{\text {average adjusted }}\right)$ exposure based on the assumption of null exposure when using a respirator and the uncorrected exposure determined by personal sampling for the rest of the day; and the background-adjusted ( $\left.C_{\text {background adjusted }}\right)$ exposure based on the assumption of null exposure when using a respirator and the background exposure determined by stationary sampling in the relevant departments.

1. Non-adjusted exposure $=C_{\text {average }}$

2. Average adjusted exposure $C_{\text {average adj }}=\left(c_{(1)} \times t_{(1)}+c_{(2)} \times\right.$ $\left.t_{(2)}\right) /\left(t_{(1)}+t_{(2)}\right)$

3. Background-adjusted exposure $C_{\text {background adj }}=\left(c_{(1)} \times t_{(1)}\right.$ $\left.+c_{(3)} \times t_{(2)}\right) /\left(t_{(1)}+t_{(2)}\right)$

$c_{(1)}=$ exposure using respirator, assuming $c_{(1)}=0$

$t_{(1)}=$ time using respirator

$c_{(2)}=$ uncorrected air concentration

$t_{(2)}=$ time spent without wearing respirator

$c_{(3)}=$ background air concentration for relevant departments

\section{Statistical analysis}

For descriptive purposes, the measured aerosol concentrations, i.e., the concentrations of inhalable, total and respirable dust, respirable quartz and cristobalite, $\mathrm{PM}_{10}$ and $\mathrm{PM}_{2.5}$, as well as the particle surface area (A-Trak) and particle number concentration (P-trak) are reported as 8-h TWAs for the full workday. Standard parameters such as arithmetic means (AM), standard deviations (SD), geometric means (GM), geometric standard deviations (GSD) and ranges were calculated based on the log-normal distributions of 
each measurement, including the stationary samples of the aerosol mass fractions and the particle number and particle surface area concentrations (Table 1). Respirable quartz exposure by job title is presented in Table 2; the corresponding descriptions, means and ranges of the measured blood concentrations by sampling day are presented in Table 3 . The analyses presented below are based on backgroundadjusted quartz and respirable particle data.

A linear mixed model was developed to describe the relationship between the different exposure metrics and the inflammatory markers. Our design and the use of mixed model made it possible to consider within- and between-worker variability based on our repeated measurements of inflammatory markers. We used a mixed model to study the variation in biomarker levels for exposure categories 1-3 (Tables 4, 5). Because the distribution of inflammatory markers was skewed, the biomarker data were log-transformed. The estimates $(\beta)$ of the fixed effects from the model allowed us to identify the factors affecting the inflammatory markers. The equation of the final mixed model is:
Table 1 Exposure concentration levels of respirable dust, quartz and cristobalite
Table 2 Exposure measurements of respirable quartz by job title

\begin{tabular}{|c|c|c|c|c|c|c|c|c|}
\hline Aerosol concentration levels & $N$ & $\mathrm{AM}$ & Median & SD & GM & GSD & Min & $\operatorname{Max}$ \\
\hline \multicolumn{9}{|l|}{ Exposure measurements } \\
\hline Respirable dust $\left(\mathrm{mg} / \mathrm{m}^{3}\right)$ & 85 & 0.85 & 0.35 & 1.6 & 0.39 & 3.1 & 0.062 & 9.7 \\
\hline Respirable quartz $\left(\mathrm{mg} / \mathrm{m}^{3}\right)$ & 85 & 0.052 & 0.018 & 0.11 & 0.016 & 4.4 & 0.0013 & 0.61 \\
\hline Respirable cristobalite $\left(\mathrm{mg} / \mathrm{m}^{3}\right)$ & 85 & 0.0037 & 0.0034 & 0.0015 & 0.0035 & 1.3 & 0.0028 & 0.014 \\
\hline \multicolumn{9}{|l|}{ Stationary measurements } \\
\hline Inhalable dust $\left(\mathrm{mg} / \mathrm{m}^{3}\right)$ & 36 & 3.2 & 2.3 & 3.9 & 1.6 & 3.9 & 0.045 & 16 \\
\hline Total dust $\left(\mathrm{mg} / \mathrm{m}^{3}\right)$ & 36 & 3.4 & 2.2 & 4.3 & 1.5 & 4.4 & 0.078 & 16 \\
\hline Respirable dust (mg/m³) & 36 & 0.58 & 0.36 & 0.58 & 0.32 & 3.3 & 0.058 & 1.9 \\
\hline Respirable quartz $\left(\mathrm{mg} / \mathrm{m}^{3}\right)$ & 36 & 0.026 & 0.011 & 0.37 & 0.031 & 6.8 & 0.0012 & 0.26 \\
\hline Respirable cristobalite $\left(\mathrm{mg} / \mathrm{m}^{3}\right)$ & 36 & 0.0034 & 0.0031 & 0.00096 & 0.0033 & 1.2 & 0.0029 & 0.0072 \\
\hline $\mathrm{PM}_{10}\left(\mathrm{mg} / \mathrm{m}^{3}\right)$ & 36 & 1.9 & 1.6 & 1.9 & 1.1 & 3.1 & 0.15 & 8.7 \\
\hline $\mathrm{PM}_{2.5}\left(\mathrm{mg} / \mathrm{m}^{3}\right)$ & 36 & 1.2 & 0.57 & 1.9 & 0.63 & 3.1 & 0.085 & 11 \\
\hline $\mathrm{PM}_{1.0}\left(\mathrm{mg} / \mathrm{m}^{3}\right)^{\mathrm{a}}$ & 35 & 0.35 & 0.2 & 0.41 & 0.27 & 3.7 & 0.018 & 1.7 \\
\hline Total particle area (A-trak) $\left(\mu \mathrm{m}^{2} / \mathrm{cm}^{3}\right)$ & 34 & 420 & 140 & 540 & 160 & 4.7 & 9.2 & 1900 \\
\hline Particle number $\left((\mathrm{P}-\right.$ trak $\left.) / \mathrm{cm}^{3}\right)$ & 36 & 78,000 & 43,000 & 91,000 & 32,000 & 4.7 & 1500 & 360,000 \\
\hline
\end{tabular}

Stationary concentration levels of inhalable, total and respirable dust, respirable quartz and cristobalite, $\mathrm{PM}_{10}, \mathrm{PM}_{2.5}, \mathrm{PM}_{1.0}$, particle surface area (A-trak) and particle number (P-trak)

$N$ number of measurements, $A M$ arithmetic mean, $S D$ standard deviation, $G M$ geometric mean, $G S D$ geometric standard deviation

${ }^{\text {a}}$ Dusttrak

\begin{tabular}{llrlllllll}
\hline Agent & Job title & N & AM & Median & SD & GM & GSD & Min & Max \\
\hline Quartz & Sand preparation & 4 & 0.026 & 0.026 & 0.018 & 0.02 & 2.4 & 0.0064 & 0.046 \\
& Melting & 13 & 0.029 & 0.013 & 0.05 & 0.013 & 3.5 & 0.0014 & 0.19 \\
& Core making & 5 & 0.09 & 0.028 & 0.14 & 0.017 & 12 & 0.0013 & 0.33 \\
& Moulding & 14 & 0.025 & 0.023 & 0.021 & 0.016 & 3.1 & 0.0014 & 0.077 \\
Casting & 4 & 0.0066 & 0.0056 & 0.004 & 0.0057 & 1.8 & 0.0029 & 0.012 \\
& Shake out & 5 & 0.12 & 0.08 & 0.11 & 0.08 & 2.8 & 0.023 & 0.29 \\
Fettling and blasting & 18 & 0.12 & 0.03 & 0.21 & 0.024 & 7.6 & 0.0014 & 0.61 \\
& Inspection & 4 & 0.029 & 0.031 & 0.024 & 0.016 & 4.6 & 0.0019 & 0.05 \\
Maintenance incl. cleaning & 4 & 0.022 & 0.024 & 0.012 & 0.019 & 2.2 & 0.0059 & 0.035 \\
& Transport & 7 & 0.016 & 0.017 & 0.0086 & 0.013 & 2 & 0.0039 & 0.029 \\
Others & 7 & 0.0066 & 0.0058 & 0.0042 & 0.0053 & 2.1 & 0.0015 & 0.014 \\
Total & 85 & 0.052 & 0.018 & 0.11 & 0.016 & 4.4 & 0.0013 & 0.61 \\
\hline
\end{tabular}

$N$ number of measurements, $A M$ arithmetic mean, $S D$ standard deviation, $G M$ geometric mean, GSD geometric standard deviation 
Table 3 Blood concentrations of biological markers (mean, median, minimum and maximum) among 85 participants on different days

\begin{tabular}{|c|c|c|c|c|c|c|c|c|c|c|}
\hline \multirow[t]{2}{*}{ Marker } & \multicolumn{5}{|c|}{ Sampling day 1} & \multicolumn{5}{|c|}{ Sampling day 2} \\
\hline & $N$ & Mean & Median & Min & Max & $N$ & Mean & Median & Min & Max \\
\hline \multicolumn{11}{|l|}{ Inflammation } \\
\hline $\mathrm{TNF} \alpha(\mathrm{pg} / \mathrm{ml})$ & 85 & 4.65 & 4.52 & 1.94 & 9.62 & 85 & 5.46 & 5.41 & 1.60 & 11.07 \\
\hline IL-1 $\beta(\mathrm{pg} / \mathrm{ml})$ & 85 & 0.20 & 0.15 & 0.02 & 0.60 & 85 & 0.35 & 0.37 & 0.02 & 0.83 \\
\hline IL-6 (pg/ml) & 85 & 1.15 & 0.95 & 0.22 & 4.25 & 85 & 1.50 & 1.26 & 0.31 & 11.20 \\
\hline IL-8 (pg/ml) & 85 & 3.23 & 2.93 & 0.76 & 10.32 & 85 & 3.48 & 3.22 & 0.85 & 7.66 \\
\hline IL-10 (pg/ml) & 85 & 0.67 & 0.61 & 0.31 & 1.52 & 85 & 0.78 & 0.75 & 0.19 & 2.30 \\
\hline IL-12 (pg/ml) & 85 & 0.74 & 0.07 & 0.07 & 3.47 & 85 & 1.10 & 0.59 & 0.07 & 12.52 \\
\hline P-SAA (mg/l) & 85 & 17.56 & 8.69 & 1.83 & 202.79 & 85 & 15.75 & 6.59 & 2.0 & 235.2 \\
\hline CRP (mg/l) & 85 & 1.98 & 1.11 & 0.08 & 13.37 & 85 & 1.82 & 0.89 & 0.04 & 14.45 \\
\hline \multicolumn{11}{|l|}{ Coagulation } \\
\hline Fibrinogen $(\mathrm{g} / \mathrm{l})$ & 85 & 3.4 & 3.4 & 1.82 & 5.06 & 85 & 3.4 & 3.37 & 1.73 & 4.88 \\
\hline D-dimer $(\mu \mathrm{g} / \mathrm{ml})$ & 85 & 0.37 & 0.35 & 0.35 & 0.90 & 85 & 0.37 & 0.35 & 0.35 & 0.76 \\
\hline vWF (kIU/l) & 85 & 1.21 & 1.12 & 0.39 & 2.47 & 85 & 1.14 & 1.04 & 0.35 & 2.34 \\
\hline Factor VIII (kIU/l) & 85 & 1.17 & 1.18 & 0.45 & 2.80 & 85 & 1.21 & 1.20 & 0.42 & 2.44 \\
\hline
\end{tabular}

\section{Results}

$$
\begin{aligned}
Y= & \ln (X)=\mu+\beta_{1}[\text { EXPOSURE }]+\beta_{2}[\mathrm{DAY}] \\
& +\beta_{3}[\mathrm{GENDER}]+\beta_{4}[\mathrm{BMI}]+\beta_{5}[\text { SMOKING }] \\
& +\beta_{6}[\mathrm{AGE}]+\beta_{7}[\text { INFECTION }] \\
& +\beta_{8}[\mathrm{BLOODGROUP}]+\varepsilon,
\end{aligned}
$$

where $Y$ is $\ln (X)$; $X$ and $Y$ are the measured and log-transformed inflammatory marker concentrations, respectively. $\mu$ is the overall average inflammatory marker concentration on the $\log$-scale and $\beta_{1}$ is the fixed effect of the exposure measure in question $(i \ldots j)$ in the given exposure group, with the lowest exposure group (exposure class 1) serving as the reference category. The exposure and stationary measurement data were categorized into three classes (with a minimum of ten workers per class) to achieve high contrast.

$\beta_{2}$ is the fixed effect of sampling time. $\beta_{3}$ is the fixed effect of gender (male or female), with males serving as the reference category. $\beta_{4}$ is the fixed effect of BMI, dichotomized by median, the highest level was used as the reference category. $\beta_{5}$ is the fixed effect of smoking habits; categories considered were smoker, ex-smoker and lifetime nonsmoker, with the latter being the reference category. $\beta_{6}$ is the fixed effect of age dichotomized by median; the oldest group served as the reference category. $\beta_{7}$ is the fixed effect of infections, with non-infection as the reference category. $\beta_{8}$ is the fixed effect of blood group (0), with other blood groups as the reference category. $\varepsilon$ is the residual.

The results of the mixed model analysis are presented as $\beta$ values and $95 \%$ confidence intervals. The statistical significance threshold was $p<0.05$. All analyses were performed with SPSS 22.0.

\section{Exposure}

The 8-h TWAs for the respirable quartz exposures measured by personal sampling of the 85 participants ranged from 0.001 to $0.61 \mathrm{mg} / \mathrm{m}^{3}$, with a mean of $0.052 \mathrm{mg} / \mathrm{m}^{3}$. In contrast, the mean and maximum quartz exposures determined by stationary measurement were $0.026 \mathrm{mg} / \mathrm{m}^{3}$ and $0.26 \mathrm{mg} /$ $\mathrm{m}^{3}$, respectively (Table 1 ). The highest personal exposures were observed in the core making, shake-out and fettling departments, for which the maxima were $0.33,0.29$ and $0.61 \mathrm{mg} / \mathrm{m}^{3}$, respectively (Table 2 ).

For the personal measurements of the 28 persons using respirators, we calculated adjusted exposure concentrations based on non-adjusted, average and background-adjusted respirable dust and quartz concentrations. The total average respirable quartz concentrations for these were $0.052,0.031$ and $0.022 \mathrm{mg} / \mathrm{m}^{3}$, respectively.

\section{Markers of inflammation and coagulation}

The measured levels of biological markers of inflammation and coagulation in the subjects' plasma and serum are presented in Table 3. These results were used in the development of the mixed model. The exposure-response analysis was based on personal exposure and stationary particle mass, number and surface area concentration measurements. Statistically significant increases in $\beta$ values (representing the fixed effects of exposure) were observed, particularly in the highest exposure group, for SAA, fibrinogen and FVIII. The particulate fractions associated with these increases were inhalable dust, $\mathrm{PM}_{2.5}$, and $\mathrm{PM}_{1}$ for SAA; inhalable dust and $\mathrm{PM}_{10}$ for FVIII; and particle number concentration 
Table 4 Mixed model for inflammatory markers IL-6, IL-8, SAA and CRP, by exposure class and antilog values

\begin{tabular}{|c|c|c|c|c|c|c|c|c|c|c|c|c|}
\hline \multirow[t]{2}{*}{ Exposure } & \multicolumn{3}{|l|}{ IL-6 } & \multicolumn{3}{|l|}{ IL-8 } & \multicolumn{3}{|l|}{ SAA } & \multicolumn{3}{|l|}{ CRP } \\
\hline & $\beta$ & \multicolumn{2}{|c|}{$95 \% \mathrm{CI}$} & $\beta$ & \multicolumn{2}{|c|}{$95 \% \mathrm{CI}$} & $\beta$ & \multicolumn{2}{|c|}{$95 \% \mathrm{CI}$} & $\beta$ & \multicolumn{2}{|c|}{$95 \%$ CI } \\
\hline \multicolumn{13}{|c|}{ Respirable quartz $\left(\mathrm{mg} / \mathrm{m}^{3} \mathrm{p}\right)$} \\
\hline$>0.05$ & 1.16 & 0.83 & 1.62 & 1.12 & 0.83 & 1.51 & 1.24 & 0.57 & 2.68 & 1.12 & 0.52 & 2.40 \\
\hline $0.01-0.05$ & 1.04 & 0.85 & 1.26 & 1.04 & 0.87 & 1.24 & 1.21 & 0.77 & 1.89 & 1.34 & 0.86 & 2.10 \\
\hline$<0.01$ & 1 & 1 & 1 & 1 & 1 & 1 & 1 & 1 & 1 & 1 & 1 & 1 \\
\hline \multicolumn{13}{|c|}{ Respirable dust (mg/m³ $\mathrm{p})$} \\
\hline$>1.0$ & 1.06 & 0.74 & 1.16 & 1.02 & 0.76 & 1.37 & 0.69 & 0.32 & 1.51 & 1.22 & 0.58 & 2.53 \\
\hline $0.1-1.0$ & 1.06 & 0.91 & 1.22 & 1.02 & 0.83 & 1.24 & 1.14 & 0.73 & 1.80 & 1.32 & 0.82 & 2.12 \\
\hline$<0.1$ & 1 & 1 & 1 & 1 & 1 & 1 & 1 & 1 & 1 & 1 & 1 & 1 \\
\hline \multicolumn{13}{|c|}{ Inhalable dust $\left(\mathrm{mg} / \mathrm{m}^{3} \mathrm{~s}\right)$} \\
\hline$>5$ & 1.37 & 0.89 & 2.12 & 1.13 & 0.77 & 1.67 & 3.22 & 1.22 & 8.47 & 2.02 & 0.73 & 5.59 \\
\hline $0.1-5$ & 1.19 & 0.84 & 1.68 & 0.86 & 0.63 & 1.17 & 1.84 & 0.91 & 3.72 & 2.06 & 0.91 & 4.65 \\
\hline$<0.1$ & 1 & 1 & 1 & 1 & 1 & 1 & 1 & 1 & 1 & 1 & 1 & 1 \\
\hline \multicolumn{13}{|c|}{ Total dust (mg/m $\left.{ }^{3} \mathrm{~s}\right)$} \\
\hline$>5$ & 1.16 & 0.84 & 1.60 & 1.24 & 0.93 & 1.64 & 2.00 & 0.91 & 4.43 & 1.05 & 0.49 & 2.24 \\
\hline $0.1-5$ & 0.96 & 0.79 & 1.17 & 0.90 & 0.75 & 1.08 & 1.12 & 0.72 & 1.73 & 0.96 & 0.59 & 1.55 \\
\hline$<0.1$ & 1 & 1 & 1 & 1 & 1 & 1 & 1 & 1 & 1 & 1 & 1 & 1 \\
\hline
\end{tabular}

Respirable dust $\left(\mathrm{mg} / \mathrm{m}^{3} \mathrm{~s}\right)$

$\begin{array}{lllllllllllll}>1.0 & 1.02 & 0.73 & 1.43 & 0.85 & 0.63 & 1.15 & 1.40 & 0.67 & 2.92 & \mathbf{1 . 4 8} & 0.68 & 3.23 \\ 0.1-1.0 & 0.97 & 0.74 & 1.28 & 0.93 & 0.72 & 1.19 & 1.48 & 0.84 & 2.59 & \mathbf{1 . 3 1} & 0.69 & 2.49 \\ <0.1 & 1 & 1 & 1 & 1 & 1 & 1 & 1 & 1 & 1 & \mathbf{1} & 1 & 1\end{array}$

Respirable quartz $\left(\mathrm{mg} / \mathrm{m}^{3} \mathrm{~s}\right)$

$\begin{array}{lllllllllllll}>0.05 & \mathbf{1 . 0 7} & 0.78 & 1.48 & 1.27 & 0.95 & 1.69 & \mathbf{1 . 8 1} & 0.83 & 3.92 & 1.01 & 0.48 & 2.11 \\ 0.01-0.05 & \mathbf{1 . 0 6} & 0.86 & 1.32 & 0.96 & 0.80 & 1.17 & \mathbf{1 . 4 8} & 0.93 & 2.34 & 1.62 & 0.99 & 2.65 \\ <0.01 & \mathbf{1} & 1 & 1 & 1 & 1 & 1 & \mathbf{1} & 1 & 1 & 1 & 1 & 1\end{array}$

$\mathrm{PM} 10\left(\mathrm{mg} / \mathrm{m}^{3} \mathrm{~s}\right)$

$\begin{array}{lllllllllllll}>2.5 & 1.42 & 0.58 & 1.34 & 0.88 & 0.58 & 1.34 & \mathbf{1 . 5 6} & 0.57 & 4.27 & 1.82 & 0.61 & 5.39 \\ 0.1-2.5 & 0.82 & 0.56 & 1.20 & 0.82 & 0.56 & 1.20 & \mathbf{1 . 5 0} & 0.62 & 3.61 & 2.03 & 0.76 & 5.44 \\ <0.1 & 1 & 1 & 1 & 1 & 1 & 1 & \mathbf{1} & 1 & 1 & 1 & 1 & 1 \\ \text { PM } 2.5\left(\mathrm{mg} / \mathrm{m}^{3} \mathrm{~s}\right) & & & & & & & & & & & \\ >2 & \mathbf{1 . 3 0} & 0.89 & 1.91 & \mathbf{1 . 2 9} & 0.92 & 1.82 & \mathbf{2 . 6 6} & \mathbf{1 . 1 1} & \mathbf{6 . 3 5} & 1.61 & 0.66 & 3.93 \\ 0.1-2 & \mathbf{1 . 1 3} & 0.86 & 1.50 & \mathbf{1 . 0 0} & 0.78 & 1.28 & \mathbf{1 . 5 4} & 0.88 & 2.69 & 1.67 & 0.87 & 3.20 \\ <0.1 & \mathbf{1} & 1 & 1 & \mathbf{1} & 1 & 1 & \mathbf{1} & 1 & 1 & 1 & 1 & 1\end{array}$

$\mathrm{PM} 1\left(\mathrm{mg} / \mathrm{m}^{3} \mathrm{~s}\right)$

$\begin{array}{lllllllllllll}>0.5 & 1.01 & 0.72 & 1.43 & 1.13 & 0.83 & 1.54 & \mathbf{2 . 5 7} & \mathbf{1 . 1 5} & \mathbf{5 . 7 3} & \mathbf{1 . 4 0} & 0.62 & 3.13 \\ 0.1-0.5 & 1.03 & 0.84 & 1.26 & 0.88 & 0.74 & 1.06 & \mathbf{1 . 2 1} & 0.78 & 1.86 & \mathbf{1 . 0 7} & 0.66 & 1.74 \\ <0.1 & 1 & 1 & 1 & 1 & 1 & 1 & \mathbf{1} & 1 & 1 & \mathbf{1} & 1 & 1\end{array}$

Particle surface area $\left(\mu \mathrm{m}^{2} / \mathrm{cm}^{3} \mathrm{~s}\right)$

$\begin{array}{lllllllllllll}>1000 & 1.19 & 0.87 & 1.64 & 1.04 & 0.79 & 1.39 & \mathbf{1 . 5 5} & 0.76 & 3.19 & \mathbf{1 . 3 5} & 0.64 & 2.84 \\ 100-1000 & 1.03 & 0.84 & 1.26 & 0.84 & 0.70 & 1.01 & \mathbf{1 . 3 3} & 0.85 & 2.08 & \mathbf{1 . 2 3} & 0.76 & 2.00 \\ <100 & 1 & 1 & 1 & 1 & 1 & 1 & \mathbf{1} & 1 & 1 & \mathbf{1} & 1 & 1\end{array}$

Particle number $\left(\mathrm{nr} / 10^{4} \mathrm{~cm}^{3} \mathrm{~s}\right)$

\begin{tabular}{lllllllllllll}
$>10$ & 1.09 & 0.85 & 1.38 & 0.83 & 0.66 & 1.03 & 1.13 & 0.68 & 1.88 & 1.20 & 0.68 & 2.12 \\
$2-10$ & 0.98 & 0.79 & 1.23 & 0.91 & 0.75 & 1.12 & 1.70 & 1.05 & 2.75 & 1.32 & 0.78 & 2.24 \\
$<2$ & 1 & 1 & 1 & 1 & 1 & 1 & 1 & 1 & 1 & 1 & 1 & 1 \\
\hline
\end{tabular}

$\beta$ fixed effect by exposure class, $95 \%$ CI (confidence interval)

Bold values indicate exposure response, both significant and nonsignificant, and italic values significant in the high exposure class

$p$ personal sampling, $s$ stationary sampling 
Table 5 Mixed model for coagulatory markers, fibrinogen, vWF, factor VIII, by exposure class and antilog values

\begin{tabular}{|c|c|c|c|c|c|c|c|c|c|}
\hline \multirow[t]{2}{*}{ Exposure } & \multicolumn{3}{|c|}{ Fibrinogen } & \multicolumn{3}{|l|}{ vWF } & \multicolumn{3}{|c|}{ Factor VIII } \\
\hline & $\beta$ & \multicolumn{2}{|c|}{$95 \% \mathrm{CI}$} & $\beta$ & \multicolumn{2}{|c|}{$95 \% \mathrm{CI}$} & $\beta$ & \multicolumn{2}{|c|}{$95 \% \mathrm{CI}$} \\
\hline \multicolumn{10}{|c|}{ Respirable quartz $\left(\mathrm{mg} / \mathrm{m}^{3} \mathrm{p}\right)$} \\
\hline$>0.05$ & 1.04 & 0.90 & 1.21 & 1.06 & 0.81 & 1.40 & 1.06 & 0.85 & 1.33 \\
\hline $0.01-0.05$ & 1.06 & 0.97 & 1.15 & 1.00 & 0.86 & 1.18 & 1.04 & 0.91 & 1.18 \\
\hline$<0.01$ & 1 & 1 & 1 & 1 & 1 & 1 & 1 & 1 & 1 \\
\hline \multicolumn{10}{|c|}{ Respirable dust $\left(\mathrm{mg} / \mathrm{m}^{3} \mathrm{p}\right)$} \\
\hline$>1.0$ & 1.10 & 0.96 & 1.26 & 0.98 & 0.77 & 1.26 & 1.11 & 0.89 & 1.39 \\
\hline $0.1-1.0$ & 1.03 & 0.94 & 1.12 & 0.94 & 0.82 & 1.07 & 1.05 & 0.91 & 1.22 \\
\hline$<0.1$ & 1 & 1 & 1 & 1 & 1 & 1 & 1 & 1 & 1 \\
\hline \multicolumn{10}{|c|}{ Inhalable dust $\left(\mathrm{mg} / \mathrm{m}^{3} \mathrm{~s}\right)$} \\
\hline$>5$ & 1.14 & 0.94 & 1.39 & 0.90 & 0.63 & 1.29 & 1.36 & 1.03 & 1.81 \\
\hline $0.1-5$ & 1.07 & 0.91 & 1.26 & 0.92 & 0.69 & 1.23 & 1.27 & 1.01 & 1.59 \\
\hline$<0.1$ & 1 & 1 & 1 & 1 & 1 & 1 & 1 & 1 & 1 \\
\hline \multicolumn{10}{|c|}{ Total dust $\left(\mathrm{mg} / \mathrm{m}^{3} \mathrm{~s}\right)$} \\
\hline$>5$ & 1.08 & 0.93 & 1.25 & 0.98 & 0.76 & 1.28 & 1.13 & 0.92 & 1.40 \\
\hline $0.1-5$ & 1.02 & 0.93 & 1.12 & 1.05 & 0.89 & 1.24 & 1.06 & 0.93 & 1.21 \\
\hline$<0.1$ & 1 & 1 & 1 & 1 & 1 & 1 & 1 & 1 & 1 \\
\hline \multicolumn{10}{|c|}{ Respirable dust (mg/m $\left./ \mathrm{m}^{3} \mathrm{~s}\right)$} \\
\hline$>1.0$ & 1.05 & 0.91 & 1.22 & 0.84 & 0.64 & 1.09 & 1.17 & 0.94 & 1.45 \\
\hline $0.1-1.0$ & 0.98 & 0.86 & 1.11 & 0.80 & 0.64 & 1.00 & 1.04 & 0.87 & 1.24 \\
\hline$<0.1$ & 1 & 1 & 1 & 1 & 1 & 1 & 1 & 1 & 1 \\
\hline \multicolumn{10}{|c|}{ Respirable quartz $\left(\mathrm{mg} / \mathrm{m}^{3} \mathrm{~s}\right)$} \\
\hline$>0.05$ & 1.04 & 0.90 & 1.20 & 1.04 & 0.80 & 1.35 & 1.13 & 0.92 & 1.40 \\
\hline $0.01-0.05$ & 1.09 & 0.99 & 1.20 & 1.08 & 0.91 & 1.29 & 1.10 & 0.96 & 1.27 \\
\hline$<0.01$ & 1 & 1 & 1 & 1 & 1 & 1 & 1 & 1 & 1 \\
\hline \multicolumn{10}{|c|}{ PM $10\left(\mathrm{mg} / \mathrm{m}^{3} \mathrm{~s}\right)$} \\
\hline$>2.5$ & 1.13 & 0.92 & 1.40 & 0.85 & 0.59 & 1.24 & 1.39 & 1.03 & 1.87 \\
\hline $0.1-2.5$ & 1.06 & 0.87 & 1.28 & 0.77 & 0.55 & 1.09 & 1.18 & 0.90 & 1.54 \\
\hline$<0.1$ & 1 & 1 & 1 & 1 & 1 & 1 & 1 & 1 & 1 \\
\hline \multicolumn{10}{|c|}{ PM $2.5\left(\mathrm{mg} / \mathrm{m}^{3} \mathrm{~s}\right)$} \\
\hline$>2$ & 1.15 & 0.97 & 1.37 & 0.90 & 0.66 & 1.23 & 1.26 & 0.98 & 1.62 \\
\hline $0.1-2$ & 1.09 & 0.96 & 1.24 & 0.92 & 0.73 & 1.15 & 1.18 & 0.98 & 1.41 \\
\hline$<0.1$ & 1 & 1 & 1 & 1 & 1 & 1 & 1 & 1 & 1 \\
\hline \multicolumn{10}{|c|}{ PM $1\left(\mathrm{mg} / \mathrm{m}^{3} \mathrm{~s}\right)$} \\
\hline$>0.5$ & 1.04 & 0.89 & 1.22 & 0.87 & 0.66 & 1.15 & 1.08 & 0.86 & 1.35 \\
\hline $0.1-0.5$ & 1.03 & 0.94 & 1.13 & 1.07 & 0.90 & 1.26 & 1.11 & 0.97 & 1.28 \\
\hline$<0.1$ & 1 & 1 & 1 & 1 & 1 & 1 & 1 & 1 & 1 \\
\hline \multicolumn{10}{|c|}{ Particle surface area $\left(\mu \mathrm{m}^{2} / \mathrm{cm}^{3} \mathrm{~s}\right)$} \\
\hline$>10$ & 1.06 & 0.92 & 1.23 & 0.93 & 0.72 & 1.21 & 1.06 & 0.86 & 1.30 \\
\hline $2-10$ & 1.09 & 1.00 & 1.20 & 1.01 & 0.85 & 1.19 & 1.10 & 0.96 & 1.26 \\
\hline$<2$ & 1 & 1 & 1 & 1 & 1 & 1 & 1 & 1 & 1 \\
\hline \multicolumn{10}{|c|}{ Particle number $\left(\mathrm{nr} / 10^{4} \mathrm{~cm}^{3} \mathrm{~s}\right)$} \\
\hline$>1000$ & 1.11 & 1.00 & 1.24 & 1.07 & 0.88 & 1.31 & 1.11 & 0.95 & 1.31 \\
\hline $100-1000$ & 1.07 & 0.97 & 1.18 & 0.96 & 0.80 & 1.15 & 1.08 & 0.93 & 1.25 \\
\hline$<100$ & 1 & 1 & 1 & 1 & 1 & 1 & 1 & 1 & 1 \\
\hline
\end{tabular}

$\beta$ fixed effect by exposure class, 95\% CI (confidence interval)

Bold values indicate exposure response, both significant and nonsignificant, and italic significant in the high exposure class

$p$ personal sampling, $s$ stationary sampling 
for fibrinogen. Non-significant exposure-responses were observed for IL-6, IL-8, SAA, CRP, vWF and FVIII with respect to respirable quartz (Tables 4,5 ).

The mixed model analysis revealed that the levels of the inflammatory and coagulatory markers TNF, IL- $1 \beta$, IL-6, IL-8 and FVIII in the second samples were significantly higher than those in the first samples, whereas the levels of SAA and vWF were significantly lower in the second samples. In addition, the levels of vWF and FVIII were significantly higher among subjects with blood group $\mathrm{O}$ than those with other blood groups. Workers reporting infections had significantly higher levels of IL-6, SAA, CRP and vWF than non-infected workers, with odds ratios of 1.6, 3.6, 3.5 and 1.5, respectively. Marker levels among smokers did not differ significantly from those among non-smokers.

\section{Discussion}

The main finding of our study is that there are significant relationships between particle mass exposure metrics and the levels of SAA, fibrinogen and FVIII. There were also nonsignificant positive exposure-response relationships between several exposure metrics and other markers of inflammation and coagulation. In particular, respirable quartz exhibited (statistically non-significant) exposure-response patterns for IL-6, IL-8, SAA, CRP, vWF and FVIII. Most of the exposure-response patterns were related to mass-based exposure metrics, but positive exposure-responses were also observed for the particle surface area and particle number concentrations. These exposure-response patterns are similar to those reported in earlier studies.

To our knowledge, these are the first published data on particle exposure (particularly, quartz exposure) and inflammatory markers among foundry workers. A key strength of this study is the use of parallel blood sampling and the determination of multiple measures of exposure, including measures based on particle mass, surface area and number. The blood sampling protocol was designed to minimize the influence of diurnal variation: sampling was only conducted at the end of a shift to enable meaningful analysis of time trends and other determinants (Rudnicka et al. 2007). The participants' blood groups were recorded because it has been suggested that exposure to occupational air pollutants may be associated with ischaemic heart disease among individuals with blood type O (Suadicani et al. 2002).

The study lacks a completely non-exposed referent group, because the use of such a group could introduce socioeconomic bias. In previous studies, we began air and blood sampling on the first day after the participants returned to work following a long vacation (Ohlson et al. 2010); this approach was not adopted here because of logistical difficulties. Our experience suggests that a single week's absence from exposed work is insufficient to establish a true baseline level of inflammatory markers (Westberg et al. 2016).

However, we believe that even without establishing such a baseline, analyses of exposure-response relationships for inflammatory markers can reveal the important effects of particle exposure at levels close to occupational exposure limits.

Particle sampling and analysis and blood sampling were performed using standard methods at accredited laboratories. The overall quartz exposures and those for specific job titles at the three foundries are comparable to previously reported exposure measurements (Andersson et al. 2009). Some $30 \%$ of the participating workers used respirators during some of their working time; the duration of these periods was recorded for each worker. Three different measures of particulate exposure were computed for these workers: the non-adjusted measure (ignoring the effect of the respirator), an adjusted measure assuming zero exposure while the respirator was worn and uncorrected exposure for the remainder of the day, and a background-adjusted measure assuming zero exposure while the respirator was worn and exposure at the background level for the worker's department otherwise. We consider the latter measure to be the most reliable. For reasons of sensitivity, mixed model analyses were performed using all three adjusted and non-adjusted measures of exposure. There were no appreciable differences between the resulting exposure-response analyses.

Workers in foundry environments may also be exposed to chemical compounds that may be linked to cardiovascular disease-notably, polycyclic aromatic hydrocarbons (PAHs) originating from incomplete combustion of organic substances, which may be emitted in the melting, casting and shake-out areas. A review of studies on PAHs in iron foundries found that the reported air concentrations of $\mathrm{B}(\mathrm{a})$ $P$ ranged from 0.01 to $2.1 \mu \mathrm{g} / \mathrm{m}^{3}$ for melting, casting and shake-out operations (Lindstedt and Sollenberg 1982).

We used log-linear modelling to study the exposure-response relationships between particle exposure and inflammatory markers. Determinants assumed to influence or confound the exposure-response relationships were included in the models, and we also determined exposure responses for subjects with and without infections, using the latter as positive controls. Workers reporting infections exhibited significantly elevated concentrations of IL-6, SAA, CRP and vWF. The observation of elevated levels of markers related to ongoing infections among this group indicates that our model design was valid for studying inflammatory responses.

CRP is one of the most sensitive acute-phase reactants and is widely used as a clinical biomarker. A review by $\mathrm{Li}$ et al. (2012) highlighted three occupational studies in which this marker was used. Among a group of 37 welders, significantly elevated levels of CRP were observed for both 
smokers and non-smokers exposed to median $\mathrm{PM}_{2.5}$ concentrations of $1.69 \mathrm{mg} / \mathrm{m}^{3}$ (25th to 75th percentile range 0.89-2.44 mg/m ${ }^{3}$ ) (Kim et al. 2005). A similar significant increase was observed among 37 steel production workers exposed to fine particles $\left(\mathrm{PM}_{1}\right.$; median exposure $0.0036 \mathrm{mg} /$ $\mathrm{m}^{3}$, range $\left.0.0017-0.033 \mathrm{mg} / \mathrm{m}^{3}\right)$ and coarse particles $\left(\mathrm{PM}_{10}\right.$; median exposure $0.162 \mathrm{mg} / \mathrm{m}^{3}$, range $0.071-1.2 \mathrm{mg} / \mathrm{m}^{3}$ ) (Bonzini et al. 2010). An analysis of 73 subjects employed in welding, cutting, grinding and in iron and aluminium foundries conducted by our group identified a statistically significant increase in CRP levels associated with a mean total dust exposure of $0.93 \mathrm{mg} / \mathrm{m}^{3}$ (range $0.034-6.4 \mathrm{mg}$ / $\mathrm{m}^{3}$ ) (Ohlson et al. 2010). Finally, a study conducted by our group on 67 workers in the pulp and paper industry showed that several mass-based exposure measures (including inhalable particulate matter, total dust and $\mathrm{PM}_{10}$ ) were associated with statistically significant increases in CRP levels, especially among the high exposure subject group. The subjects' mean inhalable dust exposure was $0.30 \mathrm{mg} / \mathrm{m}^{3}$. However, there was no detectable exposure response for the respirable fractions or the particle surface area air concentration (Westberg et al. 2016). Multiple occupational studies have thus identified increased CRP levels among workers exposed to dust concentrations of the same order of magnitude as those reported here (i.e., mean or medians between 0.1 and $1.7 \mathrm{mg} / \mathrm{m}^{3}$ ).

Fibrinogen levels exhibited exposure-response patterns with respect to all of the mass-based exposure measures other than the surface area and particle number concentrations among the foundry workers. Elevated fibrinogen levels were observed in both the high $\left(>0.05 \mathrm{mg} / \mathrm{m}^{3}\right)$ and medium $\left(0.03-0.05 \mathrm{mg} / \mathrm{m}^{3}\right)$ quartz exposure groups, and the high $(>1.0)$ and medium $\left(0.1-1.0 \mathrm{mg} / \mathrm{m}^{3}\right)$ respirable dust exposure groups. In our first study on particulate exposure in different industrial settings (Ohlson et al. 2010), we observed no exposure response for particulate matter and fibrinogen on day 1 of the investigation, but an increase was noted on day 2 . This may be because the sampling campaign in that study began immediately after the workers had returned from their summer vacation, and the fibrinogen response was comparatively slow (Saber et al. 2014). However, our study on the pulp and paper industry revealed significant exposure-response relationships between fibrinogen and several exposure measures including the inhalable dust (for which the mean concentration was $0.3 \mathrm{mg} / \mathrm{m}^{3}$, with a range of $\left.0.005-3.3 \mathrm{mg} / \mathrm{m}^{3}\right), \mathrm{PM}_{10}$, respirable dust, particle surface area and particle number concentrations (Westberg et al. 2016). Other studies have revealed significant increases in fibrinogen levels associated with high concentrations of dust containing endotoxins ( $>15 \mathrm{mg} / \mathrm{m}^{3}$ ) (Sjögren et al. 1999). However, significant reductions in fibrinogen levels have been reported among welders (Kim et al. 2005).
SAA, an acute-phase protein comparable to CRP, has not been studied in occupational environments, but there have been some studies on its responses in ambient air (Ruckerl et al. 2006). In our study on the pulp and paper industry, we observed significant increases in SAA levels (similar to those observed for CRP) associated with most mass-based exposure measures, particularly among the high exposure subject group. However, such associations were not observed for the particle surface area or particle number concentrations. SAA may play a role in atherosclerosis because it has been casually related to plaque formation in the aorta in animal studies (Vogel and Cassee 2018).

Factor VIII is a marker of inflammation and haemostasis and also a risk factor for coronary heart disease (Folsom et al. 1997; Haverkate 2002). Relations between ambient air pollutant $\left(\mathrm{PM}_{10}\right)$ (Liao et al. 2005) and wood smoke exposures (Barregård et al. 2006) and plasma levels of factor VIII have been observed previously. We found significant relations between both inhalable dust and $\mathrm{PM}_{10}$ and increases of factor VIII, indicating an increased risk of coagulation and consequently disease by this exposure.

In our first study on particle exposure during welding, cutting, grinding and other foundry operations, we identified exposure-response relationships between particulate matter and IL-6 in workers returning from long vacations who were exposed to average total dust levels of $0.93 \mathrm{mg} / \mathrm{m}^{3}$ (Ohlson et al. 2010). However, the exposure-response relationships observed for IL- 6 and other cytokines (IL-1 $\beta$, IL-10 and IL-8) in our study on the pulp and paper industry were weaker and rarer (Westberg et al. 2016). The comparative weakness of these responses can be attributed to differences in study design, particularly the fact that the exposure-free period prior to sampling was much longer in the first study than in the second (in which the exposure-free period was only 1 week long). In this work, exposure-response relationships were tentatively identified for IL- 6 and IL- 8 with respect to several mass-based exposure measures as well as the particle surface area and particle number concentrations in air.

Elevated levels of IL-6 and IL-8 were found among nanoparticle-exposed woodworkers and metalworkers when compared to a control group of office workers (Kurjane et al. 2017). Swine farmers and volunteers exposed to about $20 \mathrm{mg} / \mathrm{m}^{3}$ of organic dust for part of a work shift also showed increased levels of IL- 6 and IL- 8 , and the increase was more pronounced among the non-farmers (Palmberg et al.2002). Similar findings were obtained when volunteers were exposed to zinc oxide at $5 \mathrm{mg} / \mathrm{m}^{3}$ : the IL-6 levels induced by short-term exposure were three times higher than those observed in subjects who had experienced long-term exposure. This suggests that an adaptation or low-grade inflammatory process occurs after prolonged exposure, with comparatively low levels of cytokines (Fine et al. 2000). 
In this work, we defined three levels of quartz exposure based on our objective of studying inflammatory responses to quartz concentrations on the same order of magnitude as current occupational exposure limits. The highest exposure group considered in this work comprised individuals with exposures above $0.05 \mathrm{mg} / \mathrm{m}^{3}$, which is the exposure limit recommended by the EU (SCOEL 2002), and twice the ACGIHrecommended TWA-TLV of $0.025 \mathrm{mg} / \mathrm{m}^{3}$ (ACGIH 2018). However, these OELs are primarily intended to prevent silicosis; the limits required to minimize the risk of cardiovascular disease may be different.

Two recently published papers quantitatively examined the relationship between quartz dust exposure and cardiovascular mortality and disease. Among Chinese metal mine and pottery workers, the risk of ischaemic heart disease was almost doubled by a cumulative quartz exposure of $>0.67 \mathrm{mg} / \mathrm{m}^{3}$ year for workers exposed to $\leq 0.05 \mathrm{mg} / \mathrm{m}^{3}$ of respirable quartz. However, among workers exposed to respiratory quartz levels of $\leq 0.10 \mathrm{mg} / \mathrm{m}^{3}$, a significant risk of pulmonary heart disease occurred when the cumulative exposure exceeded $>0.86 \mathrm{mg} /$ $\mathrm{m}^{3}$ year (Liu et al. 2017). A significant increase in cardiovascular mortality was observed in a cohort of Swedish foundry workers, which was linked to respiratory quartz on the basis of an exposure-response analysis. The highest cumulative exposure to respirable quartz in this cohort was $>0.65 \mathrm{mg} / \mathrm{m}^{3}$ year (Fan et al. 2018). These cumulative exposures resulting in excess risks would correspond to a daily average exposure of around $0.02 \mathrm{mg} / \mathrm{m}^{3}$ over a period of 40 years. We observed significant exposure-response relationships between inflammatory markers and quartz levels in the highest exposure group of our cohort, whose exposure was $>0.05 \mathrm{mg} / \mathrm{m}^{3}$. Exposure at this level may thus be associated with an increased risk of cardiovascular disease.

Time series analyses of urban air pollutants indicate that cardiovascular mortality increases by $0.4 \%$ for every $10 \mu \mathrm{g} /$ $\mathrm{m}^{3}$ increase in $\mathrm{PM}_{10}$ levels (Brook et al. 2010). The geometric mean (GM) $\mathrm{PM}_{10}$ air concentration $\left(1.1 \mathrm{mg} / \mathrm{m}^{3}\right)$ determined based on our stationary measurements was used to estimate the potential increase in cardiovascular risk among our foundry workers. Assuming a background $\mathrm{PM}_{10}$ concentration of $50 \mu \mathrm{g} / \mathrm{m}^{3}$ exposure at the measured geometric mean would theoretically increase cardiovascular mortality by $42 \%$, calculated as $(1100-50) / 10 \times 0.4 \%$. This increase is of the same order as magnitude, as reported for the Swedish SMR 1.41 cohort (Fan et al. 2018).

Other chemical agents suspected to cause cardiovascular disease that are known to occur in the foundry environment include PAHs, and benzo(a)pyrene in particular (SBU 2017). Occupational exposures to these compounds are generally low when tar-free binders are used for moulding; high PAH levels typically only occur in the vicinity of melting, casting and shake-out operations. This pattern was not observed for quartz exposure levels in our study, so it is unlikely that the exposure-response patterns observed in this work can be attributed to benzo(a)pyrene exposure.

\section{Conclusion}

We have measured air exposure levels in terms of particle mass, number and surface area concentrations as well as the levels of selected inflammatory markers in workers at Swedish iron foundries, focusing on the effects of quartz dust exposure. When expressed as 8-h time-weighted averages (TWA), the average participating worker was exposed to a respirable dust concentration of $0.85 \mathrm{mg} /$ $\mathrm{m}^{3}$ (with a range of $0.062-9.7 \mathrm{mg} / \mathrm{m}^{3}$ ); the corresponding value for respirable quartz was $0.052 \mathrm{mg} / \mathrm{m}^{3}$, with a range of $0.001-0.61 \mathrm{mg} / \mathrm{m}^{3}$. Statistically significant increased levels of inflammation and coagulation markers, namely SAA, fibrinogen and FVIII, were observed in the highest exposure groups for some exposure measures. In addition, statistically non-significant exposure-response patterns were observed for respirable quartz and the markers IL-6, IL-8, SAA, CRP, vWF and FVIII. These relationships between particle exposure and inflammatory markers may indicate an increased risk of cardiovascular disease.

Acknowledgements This study was supported by the Swedish Research Council for Health, Working Life and Welfare (FORTE) Grant number 2014-0802. Alexande Hedbrant and Alexander Persson were supported by Postdoctoral Research fellowships from the Knowledge Foundation (Ref no: 20150036, ProSpekt14) and from Örebro University (Strategic Grant, Ref no: ORU 2.2.1-4060/2013).

\section{Compliance with ethical standards}

Conflict of interest The authors declare that they have no interest of conflict, commercial or non-commercial.

Informed consent Informed consent was obtained for all individual participants included in the study. The study was approved by the Regional Ethical Review Board, Uppsala, dnr. 2015/066, including the informed consent procedures.

Open Access This article is distributed under the terms of the Creative Commons Attribution 4.0 International License (http://creativeco mmons.org/licenses/by/4.0/), which permits unrestricted use, distribution, and reproduction in any medium, provided you give appropriate credit to the original author(s) and the source, provide a link to the Creative Commons license, and indicate if changes were made.

\section{References}

ACGIH (2018) Threshold limit values for chemical substances and physical agents and biological exposure indices. In: Cincinnati: American conference of governmental industrial hygienists 
American Foundry Society and Casting Industry Suppliers Association (2018) Form R-reporting of binder chemicals used in foundries. American Foundry Society, Schaumburg (ISBN 10: 0-87433-403-0)

Andersson L, Bryngelsson IL, Ohlson CG, Nayström P, Lilja BG, Westberg H (2009) Quartz and dust exposure in Swedish iron foundries. J Occup Environ Hyg 6:9-18. https://doi. org/10.1080/15459620802523943

Andjelkovich DA, Mathew RM, Richardson RB, Levine RJ (1990) Mortality of iron foundry workers: I. Overall findings. J Occup Med 32(6):529-540

Arant CB, Wessel TR, Ridker PM, Olson MB, Reis SE, Delia Johnson B, Sharaf BL, Pauly DF, Handberg E, Zineh I, Sopko G, Kelsey SF, Noel Bairey Merz C, Pepine CJ (2009) Multimarker approach predicts adverse cardiovascular events in women evaluated for suspected ischemia: results from the National Heart, Lung, and Blood Institute-sponsored Women's Ischemia Syndrome Evaluation. Clin Cardiol 32:244-250

Barregård L, Sällsten G, Gustafson P, Andersson L, Johansson L, Basu S, Stigendal L (2006) Experimental exposure to wood-smoke particles in healthy humans: effects on markers of inflammation, coagulation, and lipid peroxidation. Inhal Toxicol 18(11):845-853

Bonzini M, Tripodi A, Artoni A, Tarantini L, Marinelli B, Bertazzi PA, Apostoli P, Baccarelli A (2010) Effects of inhalable particulate matter on blood coagulation. J Thromb Haemost 8:662-668

Brook RD, Rajagopalan S, Pope CA 3rd, Brook JR, Bhatnagar A, Diez-Roux AV, Holguin F, Hong Y, Luepker RV, Mittleman MA, Peters A, Siscovick D, Smith SC Jr, Whitsel L, Kaufman JD (2010) American Heart Association Council on Epidemiology and Prevention, Council on the Kidney in Cardiovascular Disease, and Council on Nutrition, Physical Activity and Metabolism Particulate matter air pollution and cardiovascular disease: an update to the scientific statement from the American Heart Association. Circulation 121:2331-2378

Dagenais M, Skeldon A, Saleh M (2012) The inflammasome: in memory of Dr. Jurg Tschopp. Cell Death Differ 19:5-12

Danesh J, Whincup P, Walker M, Lennon L, Thomson A, Appleby P, Gallimore JR, Pepys MB (2000) Low grade inflammation and coronary heart disease: prospective study and updated meta-analyses. BMJ 321:199-204

Danesh J, Kaptoge S, Mann AG, Sarwar N, Wood A, Angleman SB, Wensley F, Higgins JPT, Lennon L, Eiriksdottir G, Rumley A, Whincup PH, Lowe GDO, Gudnason V (2008) Long-term interleukin-6 levels and subsequent risk of coronary heart disease: two new prospective studies and a systematic review. PLoS Med 5: e78. http://www.plosmedicine.org. Accessed Dec 312018

Decoufle P, Wood DJ (1979) Mortality patterns among workers in a gray iron foundry. Am J Epidemiol 109:667-675

Danesh J, Lewington S, Thompson SG, Lowe GD, Collins R, Kostis JB, Wilson AC, Folsom AR, Wu K, Benderly M, Goldbourt U, Willeit J, Kiechl S, Yarnell JW, Sweetnam PM, Elwood PC, Cushman M, Psaty BM, Tracy RP, Tybjaerg-Hansen A, Haverkate F, de Maat MP, Fowkes FG, Lee AJ, Smith FB, Salomaa V, Harald K, Rasi R, Vahtera E, Jousilahti P, Pekkanen J, D'Agostino R, Kannel WB, Wilson PW, Tofler G, Arocha-Piñango CL, RodriguezLarralde A, Nagy E, Mijares M, Espinosa R, Rodriquez-Roa E, Ryder E, Diez-Ewald MP, Campos G, Fernandez V, Torres E, Marchioli R, Valagussa F, Rosengren A, Wilhelmsen L, Lappas G, Eriksson H, Cremer P, Nagel D, Curb JD, Rodriguez B, Yano K, Salonen JT, Nyyssönen K, Tuomainen TP, Hedblad B, Lind P, Loewel H, Koenig W, Meade TW, Cooper JA, De Stavola B, Knottenbelt C, Miller GJ, Cooper JA, Bauer KA, Rosenberg RD, Sato S, Kitamura A, Naito Y, Palosuo T, Ducimetiere P, Amouyel P, Arveiler D, Evans AE, Ferrieres J, Juhan-Vague I, Bingham A, Schulte H, Assmann G, Cantin B, Lamarche B, Després JP, Dagenais GR, Tunstall-Pedoe H, Woodward M, Ben-Shlomo Y,
Davey Smith G, Palmieri V, Yeh JL, Rudnicka A, Ridker P, Rodeghiero F, Tosetto A, Shepherd J, Ford I, Robertson M, Brunner E, Shipley M, Feskens EJ, Kromhout D, Dickinson A, Ireland B, Juzwishin K, Kaptoge S, Lewington S, Memon A, Sarwar N, Walker M, Wheeler J, White I, Wood A, Fibrinogen Studies Collaboration (2005) Plasma fibrinogen level and the risk of major cardiovascular diseases and nonvascular mortality: an individual participant meta-analysis. JAMA 294:1799-1809

Dostert C, Pétrilli V, Van Bruggen R, Steele C, Mossman BT, Tschopp J (2008) Innate immune activation through Nalp3 inflammasome sensing of asbestos and silica. Science 320(5876):674-677

European standard: EN 12341 (1999) Air quality-determination of the PM10 fraction of suspended particulate matter-reference method and field test procedure to demonstrate reference equivalence of measurement methods

European standard: EN14907 (2005) Standard gravimetric measurement method for the determination of PM2.5 mass fraction of suspended particulate matter in ambient air

Fan C, Graff P, Vihlborg P, Bryngelsson IL, Andersson L (2018) Silica exposure increases the risk of stroke but not myocardial infarction-a retrospective cohort study. PLoS One 13(2):e0192840. https://doi.org/10.1371/journal.pone.0192840

Fine JM, Gordon T, Chen LC, Kinney P, Falcone G, Sparer J, Beckett WS (2000) Characterization of clinical tolerance to inhaled zinc oxide in naive subjects and sheet metal workers. J Occup Environ Med 42:1085-1091

Folsom AR, Wu KK, Rosamond WD, Sharrett AR, Chambless LE (1997) Prospective study of hemostatic factors and incidence of coronary heart disease: the atherosclerosis risk in communities (ARIC) Study. Circulation 96(4):1102-1108

Haverkate F (2002) Levels of haemostatic factors, arteriosclerosis and cardiovascular disease. Vasc Pharmacol 39(3):109-112

Health and Safety Executive (HSE 2000) (2000) MDHDS: general methods for sampling and gravimetric analysis of inhalable and respirable dust. Report no 14/3, February 2000, Suffolk UK

Kawasaki H (2015) A mechanistic review of silica-induced inhalation toxicity. Inhal Toxicol 27(8):363-377. https://doi. org/10.3109/08958378.2015.1066905 (Epub 2015 Jul 21)

Kim JY, Chen J-C, Boyce PD, Christiani DC (2005) Exposure to welding fumes is associated with acute systemic inflammatory responses. Occup Environ Med 62:157-163

Koskela R, Mutanen P, Sorsa JA, Klockars M (2005) Respirarory disease and cardiovascular disease. Occup Environ Med 62:650-665

Kurjane N, Zvagule T, Reste J, Martinsone Z, Pavlovska I, Martinsone I, Vanadzins I (2017) The effect of different workplace nanoparticles on the immune systems of employees. J Nanopart Res 19(9):320. https://doi.org/10.1007/s11051-017-4004-6 (Epub 2017 Sep 13)

Li Y, Rittenhouse-Olson K, Scheider WL, Mu L (2012) Effect of particulate matter air pollution on C-reactive protein: a review of epidemiologic studies. Rev Environ Health 27:133-149

Liao D, Heiss G, Chinchilli VM, Duan Y, Folsom AR, Lin HM, Salomaa V (2005) Association of criteria pollutants with plasma hemostatic/inflammatory markers: a population-based study. J Expo Anal Environ Epidemiol 15(4):319-328

Lindstedt G, Sollenberg J (1982) Polycyclic aromatic hydrocarbons in the occupational environment: with special reference to benzo[a] pyrene measurements in Swedish industry. Scand J Work Environ Health 8(1):1-19 (Review)

Liu Y, Zhou Y, Hnizdo E, Shi T, Steenland K, He X, Chen W (2017) Total and cause-specific mortality risk associated with low-level exposure to crystalline silica: a 44-Year cohort study from China. Am J Epidemiol 186(4):481-490. https://doi.org/10.1093/aje/ kwx124

Nayström P (2018) Annual summary of the Swedish Foundry industry 2016 personal communication 
NIOSH (1994a) Manual of analytical methods, 4th edition. Silica, crystalline, by XRD: method 7500 In: NIOSH Manual of Analytical Methods, 4th ed. DHHS (NIOSH) Pub. No. 94-113. NIOISH. Cincinnati, $\mathrm{OH}$

NIOSH (1994b) Manual of analytical methods, 4th edition. Particulates not otherwise regulated: method 0500 In: NIOSH Manual of Analytical Methods, 4th ed. DHHS (NIOSH) Pub. No. 94-113. NIOISH. Cincinnati, OH

Ohlson CG, Berg P, Bryngelsson IL, Elihn K, Ngo Y, Westberg H, Sjögren B (2010) Inflammatory markers and exposure to occupational air pollutants. Inhal Toxicol 22:1083-1090

Palda VA (2003) Is foundry work a risk for cardiovascular disease? A systematic review. Occup Med (Lond) 53(3):179-190

Palmberg L, Larssson BM, Malmberg P, Larsson K (2002) Airway responses of healthy farmers and nonfarmers to exposure in a swine confinement building. Scand J Work Environ Health 28(4):256-263

Peeters PM, Perkins TN, Wouters EF, Mossman BT, Reynaert NL (2013) Silica induces NLRP3 inflammasome activation in human lung epithelial cells. Part Fibre Toxicol 12:10-13

Rees D, Weiner R (1994) Dust and pneumoconiosis in the South African foundry industry. S Afr Med J 84(12):851-855

Rodriguez V, Tardon A, Kogevinas M, Prieto CS, Cueto A, Garcia M, Menendez IA, Zaplana J (2000) Lung cancer risk in iron and steel foundry workers: a nested case control study in Asturias, Spain. Am J Ind Med 38(6):644-650

Rosenman KD, Reilly MJ, Rice C, Hertzberg V, Tseng CY, Anderson HA (1996) Silicosis among foundry workers. Implication for the need to revise the OSHA standard. Am J Epidemiol 144(9):890-900

Ruckerl R, Ibald-Mulli A, Koening W, Schneider A, Woelke G, Cyrys J, Heinrich J, Marder V, Frampton M, Wichmann HE, Peters A (2006) Air pollution and markers of inflammation and coagulation in patients with coronary heart disease. Am J Respir Crit Care Med 173:432-441

Rudnicka AR, Rumley A, Lowe GDO, Strachan DP (2007) Diurnal, seasonal, and blood-processing patterns in levels of circulating fibrinogen, fibrin D-dimer, C-reactive protein, tissue plasminogen activator and von Willebrand factor in a 45 year-old population. Circulation 115:996-1003

Saber AT, Jacobsen NR, Poulsen SS, Kyjovska ZO, Halappanavar S, Yauk CL, Wallin H, Vogel U (2014) Particle -induced pulmonary acute phase response may be the link between particle inhalation and cardiovascular disease. Wiley Interdiscip Rev Nanomed Nanobiotechnol 6(6):517-531

SBU (2017) Occupational health and safety-chemical exposure. Report 261. http://www.sbu.se/en/publications/sbu-assesses/ occupational-health-and-safety-chemical-exposure/. Accessed Dec 312018

Schudel W (1960) Studies on the relation between foundry silicosis and tuberculosis. Arch Gewerbepathol Gewerbehyg 17:643-650
SCOEL (2002) Recommendations from Scientific Committee on Occupational Exposure Limits for silica, crystalline (respirable dust). Report no:SCOEL/SSUM/94 final." Scientific Committee on Occupational Exposure Limits, Brussels, p 13

Seaton A, MacNee W, Donaldson K, Goddon D (1995) Particulate air pollution and acute health effects. Lancet 345:176-178

Sherson D, Svane O, Lynge E (1991) Cancer incidence among foundry workers in Denmark. Arch Environ Health 46(2):75-81

Silverstein M, Maizlich N, Park R, Broddsky L, Mirer F, Silverstein B, Brodsky L, Mirer F (1986) Mortality among ferrous foundry workers. Am J Ind Med 10:27-43

Sjögren B (1997) Occupational exposure to dust: inflammation and ischaemic heart disease. Occup Environ Med 54:466-469

Sjögren B, Wang Z, Larsson B-M, Larsson K, Larsson PH, Westerholm $P$ (1999) Increase in interleukin-6 and fibrinogen in peripheral blood after swine dust inhalation. Scand J Work Environ Health 25:39-41

Suadicani AT, Hein HO, Gynteberg F (2002) Airborne occupational exposure, $\mathrm{ABO}$ phenotype and risk of ischemic heart disease in the Copenhagen Male Study. J Cardiovasc Risk 9(4):191-198

Vena JE, Sultz HA, Fiedler RC, Barnes A (1985) Mortality of workers in an automobile engine and parts manufacturing complex. $\mathrm{Br} \mathrm{J}$ Ind Med 42:85-93

Vincent J (2005) Health- related aerosol measurement: a review of existing sampling criteria and proposals for new ones. J Environ Monit 7:1037-1053

Vogel U, Cassee FR (2018) Editorial: dose-dependent ZnO particleinduced acute phase response in humans warrants re-evaluation of occupational exposure limits for metal oxides. Part Fibre Toxicol 15(1):7. https://doi.org/10.1186/s12989-018-0247-3

Westberg HB, Bellander T (2003) Epidemiological adaptation of quartz exposure modeling in Swedish aluminum foundries: nested case-control study on lung cancer. Appl Occup Environ Hyg 18(12):1006-1013

Westberg H, Elihn K, Andersson E, Persson B, Andersson L, Bryngelsson IL, Karlsson C, Sjögren B (2016) Inflammatory markers and exposure to airborne particles among workers in a Swedish pulp and paper mill. Int Arch Occup Environ Health 89(5):813-822. https://doi.org/10.1007/s00420-016-1119-5 (Epub 2016 Feb 13)

Xu Z, Brown LM, Pan GW, Liu TF, Gao GS, Stone BJ et al (1996) Cancer risks among iron and steel workers in Anshan, China, part II: case-control studies of lung and stomach cancer. Am J Ind Med 30(1):7-15

Yoon JH, Hahn YE (2014) Cause specific mortality due to malignant and non-malignant disease in Korean foundry workers. PLoS One 9(2):e8836413(2)

Publisher's Note Springer Nature remains neutral with regard to jurisdictional claims in published maps and institutional affiliations. 